\title{
Near Realtime Searches for Neutrinos from GRBs with IceCube
}

\author{
The IceCube Collaboration \\ ${ }^{\dagger}$ http://icecube.wisc.edu/collaboration/authors/icrc15_icecube \\ E-mail: jfelde@icecube.umd.edu
}

\begin{abstract}
Gamma Ray Bursts (GRBs) have long been suspected as the sources for the ultra high-energy cosmic rays. For this to be true, a mechanism must exist within the GRB to produce hadrons, a consequence of which is the production of neutrinos. So far, no significant observation has been made that suggests GRBs produce neutrinos. The IceCube neutrino Observatory, a cubic kilometer ice Cherenkov detector at the South Pole has put stringent limits on the theoretical models predicting prompt GRB neutrino and cosmic ray production. Although no significant observation has yet been made, even a single high-energy neutrino event coincident in time and direction to a known GRB could constitute a discovery. As such, we have implemented a rapid data processing and analysis scheme that allows for detection of possible GRB coincident neutrino events, as well as for the ability to send alerts of such events to other observatories for follow up observations. In this poster we describe the data processing and analysis scheme, the sensitivity of current searches, and the mechanism in place to send alerts for future IceCube observations.
\end{abstract}

Corresponding authors: J. Felde*1

${ }^{1}$ University of Maryland, College Park, MD 20742-4111

The 34th International Cosmic Ray Conference,

30 July- 6 August, 2015

The Hague, The Netherlands

${ }^{*}$ Speaker. 


\section{Introduction}

Gamma Ray Bursts (GRBs) are known astrophysical phenomena that release a tremendous amount of energy, approximately $10^{51}-10^{54} \mathrm{erg} \times \Omega / 4 \pi$, where $\Omega$ is the solid angle of a possible beamed emission, in a short amount of time, usually less than a couple of minutes [1]. Due to the extreme energy available, the possibility that GRBs are the sources of ultra high-energy cosmic rays (UHECRs) with energies above $10^{18} \mathrm{eV}$ seems plausible, assuming a mechanism exists within the GRB acceleration engine to accelerate protons with a similar efficiency as electrons. Since the protons are charged, their trajectories will be altered by galactic and intergalactic magnetic fields, ruining any space and time correlation to their source. The presence of protons in the acceleration engine would give rise to interactions such as $p+\gamma \rightarrow \Delta^{+} \rightarrow n+\pi^{+}$, where the pion would subsequently decay producing muon and electron neutrinos. Neutrinos are neutral, fundamental particles that are only weakly interacting, and as such, are capable of propagating over cosmic distances undeflected. If neutrinos are detected in spatial and temporal correlation with a known GRB, it would confirm the existence of high-energy proton acceleration in GRBs, supporting the hypothesis that GRBs produce the observed UHECRs.

In 2013, IceCube announced a discovery of high-energy neutrinos, above $10 \mathrm{TeV}$, of extraterrestrial origin, but no sources have been identified so far [2]. A recent search for neutrinos on time and on source from known GRBs during three years of IceCube construction (40, 59, and 79 strings) and one year with the completed (86 string) detector showed no significant correlation and is able to place tight limits on prompt neutrino production from GRBs. We conclude that no more than about $1 \%$ of the measured astrophysical flux my be attributed to prompt emission from known GRBs. Furthermore, tight limits can be placed on models that predict neutrino fluxes from GRBs; these results will be discussed in section 7. Although it does not appear that GRBs are ultimately responsible for the UHECRs, neutrinos may still be produced by GRBs. Moving forward, IceCube plans to automate previous analyses to search for neutrinos coincident with GRBs with short latency and contribute to the Gamma-ray Coordinate Network (GCN) by submitting notices when candidate events are detected [3]. The mechanism for such alerts is outlined in section 8 .

\section{IceCube}

IceCube is a cubic kilometer scale ice Cherenkov detector instrumented in the polar ice cap at the South Pole. The full detector, completed in December of 2010, consists of 5160 optical modules (DOMs) deployed along 86 vertical strings. The active volume begins about $1.5 \mathrm{~km}$ below the surface to shield low energy cosmic ray muons. High-energy charged leptons produce Cherenkov radiation as they propagate through the ice. This radiation may be detected by the DOMs, each of which contains a 10-inch photomultiplier tube that converts the collected radiation into an amplified analog pulse that is subsequently digitized on board and sent to the data acquisition (DAQ) system on the surface where the trigger conditions are applied. The triggered events are then processed and filtered at the South Pole. A number of fast reconstructions are applied to the events before the data are saved and transferred to the north via a TDRSS satellite link. 


\section{Event Reconstructions}

Triggered events passing simple event classification filters are reconstructed online using a maximum likelihood method [4]. The reconstruction fits the temporal and spatial topologies of the DOMs that detected a signal in each event. For this analysis, only upward going muon track events are considered for which a neutrino pure signal is attainable due to the Earth's shielding of cosmic ray muons. The region beyond the horizon, down to a declination of $-5^{\circ}$, is also included because the ice cap is thick enough in this region to provide sufficient overburden.

The parameters used for event selection are described in section 4. Of primary importance to the analysis are the energy and angular reconstructions. From simulation we have determined that the angular resolution on track events is better than $1^{\circ}$ for energies greater than $3 \mathrm{TeV}$. At the energies that IceCube is sensitive to, the relativistic boost factor is large and the neutrino and muon are highly collinear, such that the reconstructed muon vector can be used to point back in the direction of the incoming neutrino. An estimate of the reconstructed energy may be obtained by measuring the total charge deposited to all DOMs during an event. This energy estimate can only provide a lower bound on the true neutrino energy since a portion of the incoming and/or outgoing track is typically not contained. Assuming an $\mathrm{E}^{-2}$ spectrum, the median energy error is a factor of 4 times the reconstructed energy. Nevertheless, the steeply falling energy distribution of the background flux renders the energy estimate useful for distinguishing background from signal.

\section{Event Selection}

Despite searching for upward going neutrinos, the dominant background at trigger level is due to downward going cosmic ray muons which trigger IceCube at a rate of about $2.5 \mathrm{kHz}$. Most of these muons will be properly reconstructed as downward going and removed from the analysis, but some downward going muons are mis-reconstructed as upward going. Muons passing near the boundary of the instrumented volume can produce light patterns that propagate upwards, and coincident cosmic ray muons can also leave light patterns that reconstruct as upward going. Both cases are well distinguished from true upward going muons using fit quality, fit stability, and event topology parameters. After loose pre-cuts that mostly remove poorly reconstructed events, 13 parameters are used to train Boosted Decision Tree forests using well reconstructed simulated neutrinos as signal and off-time (not within \pm 2 hours of a GRB) data as background.

An irreducible background of atmospheric neutrinos will remain in the sample at final cut level. On time atmospheric neutrinos are indistinguishable from neutrinos produced by GRBs, and can only be probabilistically differentiated based on their energy and direction with respect to a known GRB. The final cut level is optimized for discovery potential independently for each detector configuration. At this level, well reconstructed simulated neutrinos following an $\mathrm{E}^{-2}$ spectrum have a greater than $80 \%$ efficiency with respect to trigger level, and yield a final event rate of less than $4 \mathrm{mHz}$.

\section{GRB Selection}

Notices and circulars reported to the GRB Coordinates Network (GCN) [3] by satellite experiments are parsed and stored by an automated system called GRBWeb [5]. GRBWeb is available on 
the web and provides per burst information from the experiments reporting GRB detection. When multiple satellites observe the same burst, the time window is defined by the most inclusive start and stop times reported. The angular position and error is given by the satellite with the smallest reported angular error, and the spectral parameters of the gamma ray fluence are preferentially taken from Fermi GBM [6] [7], Konus-Wind [8], Suzaku [9], Swift/BAT [10], and INTEGRAL [11], in that order. The gamma ray fluence parameters are used for modeling the neutrino fluence predictions. When a parameter is not reported, an average value is used, where the average values are calculated separately for short bursts (less than $2 \mathrm{~s}$ ) and long bursts (greater than $2 \mathrm{~s}$ ).

\section{Analysis}

The analysis presented here is designed to discover a neutrino signal arriving from the direction of a known GRB coincident in time with the gamma ray signal observed by a satellite experiment. Neutrino emission models that do not adhere to this paradigm are not well constrained by such an analysis. To determine the level at which the observed data supports the hypothesis that GRBs produce neutrinos, an unbinned likelihood analysis is used. The underlying likelihood function is a ratio of signal like to background like probability density functions (PDFs) that describe the relative probabilities of observing a signal or background event as a function of reconstructed event energy, event time with respect to the observed gamma ray emission time, and the event direction with respect to the observed GRB location. It is denoted as $S / B=(S / B)_{\text {energy }} \times(S / B)_{\text {time }} \times(S / B)_{\text {space }}$.

To calculate the $(S / B)_{\text {energy }}$ term, the reconstructed energies of simulated neutrino events, weighted to an $\mathrm{E}^{-2}$ spectrum, are used to describe the signal PDF. The background energy PDF is taken from off-time data. Beyond $100 \mathrm{TeV}$, the low number of observed data events start to add statistical uncertainty to the PDF ratio. To remove the effects of this uncertainty at high energy, the simulated neutrino sample is weighted to an atmospheric neutrino flux model and used to extend the background energy PDF beyond $10 \mathrm{PeV}$.

The $(S / B)_{\text {time }}$ term is calculated based on the time difference between the observed event time and the GRB start time, $\Delta t=\left(t_{\mathrm{event}}-t_{\mathrm{GRB}}\right)$. The signal time PDF is constructed to have constant probability during the gamma detection duration. Gaussian tails are appended prior to the start, and following the end of the burst duration. The width of the appended Gaussian functions is given by the burst duration, where a minimum of $2 \mathrm{~s}$ and maximum of $30 \mathrm{~s}$ is imposed if the burst duration is outside of this range. This treatment preserves some sensitivity to precursor and afterglow periods. The Gaussians are normalized such that there is a smooth transition at the boundaries of the burst start and end times. To lower processing time, the tails of the signal time PDF are truncated at four times the width $(4 \sigma)$ so that events outside this window automatically receive a signal PDF equal to zero, and therefore need not be considered. A constant value is used for the background time PDF and is defined over the same interval for which the signal time PDF is non-zero.

Finally, the term $(S / B)_{\text {space }}$ is constructed from $\Delta \Psi$, the opening angle between the reconstructed event direction and observed GRB direction vectors, along with the associated errors on those directions. A recent modification to the analysis is the use of the Kent distribution [12], a probability density function normalized on the unit sphere which provides a better description to the spread in angular directions than the previously used circularized two dimensional Gaussian. Assuming circularized errors, such that the contours of equal probability on the sphere are circular, 
the Kent distribution simplifies to $f(\Delta \Phi, \kappa)=(\kappa / 4 \pi \sinh (\kappa)) \times \exp (\kappa \cos (\Delta \Phi))$, where $\kappa$ sets the spread of the distribution and is taken to be $\kappa=1 /\left(\sigma_{\mathrm{GRB}}^{2}+\sigma_{\text {event }}^{2}\right)$. This new treatment has a very small impact on the muon track analysis because the event and GRB angular errors are typically well below about $20^{\circ}$ when the difference between the Kent and Gaussian treatments becomes noticeable in the tails. For consistency, however, this treatment was adopted because it is necessary for complementary analyses that accept cascade like events from $v_{\mathrm{e}}$ and $v_{\tau}$ charged current interactions as well as neutral current interactions. These events typically have poor pointing resolution, often greater than $20^{\circ}$. The background space PDF is determined from off-time data and accounts for the geometrical efficiencies of the detector from various directions.

For an ensemble of events, the PDFs can be used to evaluate how signal or background like the events are. The following test statistic is used to encapsulate the degree to which an ensemble of $\mathrm{N}$ events is signal like or background like:

$$
T=-n_{s}+\sum_{i=1}^{N} \ln \left(\frac{n_{s} S_{i}}{\left\langle n_{b}\right\rangle B_{i}}+1\right),
$$

which is the logarithm of the likelihood that the ensemble represents $n_{s}+\left\langle n_{b}\right\rangle$ signal plus background events, divided by the likelihood that the ensemble represents only $\left\langle n_{b}\right\rangle$ background events and no signal events. The value of $n_{s}$ is chosen such that it maximizes this log likelihood ratio for the given event ensemble. So far, IceCube has yet to observe a statistically significant neutrino event on time and on source with a GRB, and so limits are placed on various neutrino production models. The best limits are derived by "stacking" the GRB time windows into one single exposure. The value of $\left\langle n_{b}\right\rangle$ is then determined for the entire exposure under consideration. In the future, we will also evaluate $T$ over the time window of each GRB. For such per GRB test statistics, the value of $\left\langle n_{b}\right\rangle$ is much smaller due to the smaller time window.

The significance of an observed $T$ value, $T_{\mathrm{obs}}$, is obtained from a comparison against the distribution of $T$ assuming that only background events are present. For many trials, pseudo events are selected based on the background energy and space PDFs from the off-time data. Arbitrary signal models can be tested by injecting not only background events, but also signal like events from simulation which are drawn according to the signal energy, space, and time PDFs, where each event carries a weight determined by the specific signal model under consideration. The weight for each signal event is equivalent to the probability that such an event should be injected. For a given signal model, the signal strength is adjusted while evaluating a sufficiently large number of random trials. Following the Feldman-Cousins prescription [13], a 90\% confidence upper limit is determined by the signal strength required such that $90 \%$ of trials yield a $T$ greater than $T_{\mathrm{obs}}$.

\section{Recent Results}

The latest results from IceCube are based on the analysis of four years of data, three years while under construction and one year with the completed detector, and no significant correlation between known GRBs and upward going neutrino events was found [14]. A single event was found that correlates to an observed GRB, however, even though the event was on time, it was not within the $1 \sigma$ angular error circle, and had a relatively modest muon reconstructed energy of $10 \mathrm{TeV}$. Combining all of the GRBs into a single stacked exposure yields a test statistic, $T=0.1330$ which 
corresponds to a significance of $p=0.33$. In lieu of a signal discovery, these results can be used to improve the limits on various GRB neutrino production models. A general class of models exists where neutrinos are produced via $\mathrm{p} \gamma$ interactions in a GRB fireball. Assuming that GRBs are the dominant sources of cosmic rays observed at Earth, these models predict a doubly-broken power law spectrum in the Earth's frame. This total quasi-diffuse flux is given by:

$$
\Phi_{v}(E)=\Phi_{0} \cdot\left\{\begin{array}{lr}
E^{-1} \varepsilon_{b}^{-1} & E<\varepsilon_{b}, \\
E^{-2} & \varepsilon_{b} \leq E<10 \varepsilon_{b}, \\
E^{-4}\left(10 \varepsilon_{b}\right)^{2} & 10 \varepsilon_{b} \leq E
\end{array}\right.
$$

Exclusion contours are shown in Figure 1. Also shown on the plot are two representative model predictions assuming prompt neutrino emission. The first assumes that only neutrons escape from the GRB fireball to contribute to the UHECRs [15]. This scenario predicts a large neutrino flux, and is strongly excluded by the limits. The second prediction, the Waxman-Bahcall model, allows for direct proton escape from the GRB fireball and hence can account for the UHECRs without requiring a large neutrino flux [16]. We do not yet fully exclude this

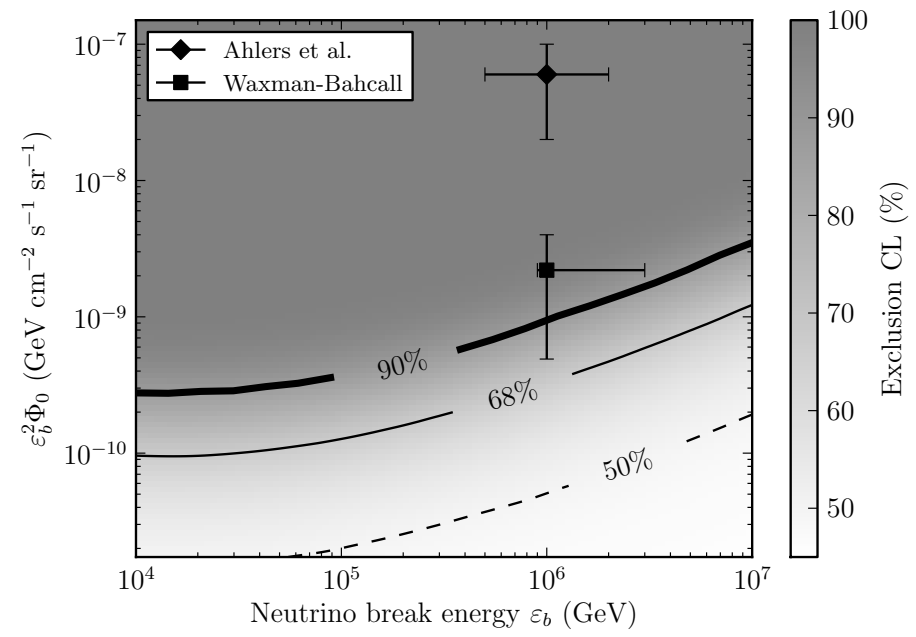

Figure 1: Exclusion regions for a generic double broken power law $v$ flux models as a function of the spectrum break energy, $\varepsilon_{\mathrm{b}}$, and flux normalization, $\Phi_{0}$. scenario at the $90 \%$ confidence level. More details on the results are available in reference [14].

\section{Near Realtime Alerts}

In view of the recent results, the current limits already place tight constraints on typical model assumptions for prompt neutrino production in GRBs. In the near future, data from three additional years taken with the completed IceCube detector will be included and searches for cascade like events as well as muon tracks originating from the southern sky will be considered. Whether a signal is present or not, we wish to maximize our chances for a discovery. In pursuit of this goal, a near realtime data processing system has been created to allow IceCube to further contribute to the multi-messenger strategy for studying GRBs.

Due to the logistical challenges of working from the South Pole, a processing pipeline has been developed in the north to process IceCube data as quickly as possible. Although computer resources are abundant outside of the South Pole, a significant delay is suffered due to the existing data transfer procedures which rely on a transfer link via TDRSS satellite. Data taken by IceCube is typically not available elsewhere for at least about one day after the data was taken. Once the data is available, the processing pipeline is very fast and adds minimally to the total latency. Figure 
2 shows the observed latency of this system for the time period just prior to submitting these proceedings. It is evident that the typical latency of this system is between one and two days, as expected.

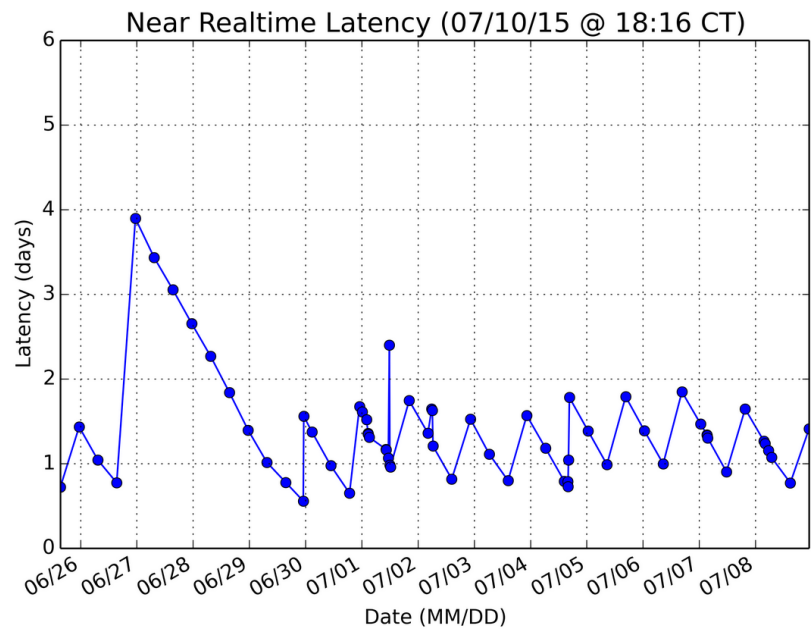

Figure 2: Observed latency of IceCube data with the northern track GRB event selection applied. The Latency is dominated by delay of daily TDRSS transfer link and is typically in the range of one to two days; occasionally it will increase for a few days.
For the northern track analysis, all necessary reconstruction variables are immediately available as they are calculated online at the South Pole. Events which pass the event selection are stored locally and compared to a running GRB sample compiled from GRBweb every 10 minutes. In some cases, the GRB information may lag behind the neutrino data. Therefore, the analysis is triggered both when new events are processed, and when new GRB information is available. For future southern track and cascade analyses, the event selection requires additional computation time that makes it unfeasible to select events in realtime. The possibility does exist, however, to trigger such searches when a new GRB

is observed. Such a mechanism is under development, but it is expected that the northern track analysis will be the first implemented by this system.

The analysis of events will follow the per-burst description provided in Section 6. The standard signal and background PDFs will be used to evaluate the test statistic only including the time window when the signal time PDF is non-zero. The significance for a single coincident event will be larger in the per-burst case because the average expected background rate is suppressed by the short search duration. The per-burst calculation is also more sensitive to multiplet signals where two or more events are seen on time with a GRB. A single high-energy on time on source event, or two events with modest energy, could very well constitute a discovery.

If the per-burst analysis returns a zero test statistic, no alert is generated. If a non-zero test statistic is returned, an automated alert will be generated and submitted to GCN as a notice. If further information is either needed or available, the notice will be followed up by a circular that details the event(s) contributing to the alert. There is also a desire to archive a subset of the neutrino events with the Astrophysical Multimessenger Observatory Network (AMON) for use in additional correlation studies of a possible simultaneous detection of sub discovery threshold events among two or more contributing observatories [17]. 


\section{Conclusion}

IceCube has searched for upward going muon neutrinos in coincidence with GRBs detected by satellite observatories. So far no significant detection has been made and the absence of a signal is used to place strong limits on models predicting prompt neutrino production in GRB fireballs. Many additional models exist which are not explicitly tested by current analyses. A web based tool was developed for public use that allows anyone to derive limits to their chosen model based on the four year IceCube northern track search. The link is available in reference [18]. As IceCube continues, a near realtime (latency of one to two days) data processing and alert system will soon be implemented to quickly search for a coincident neutrino signal from newly observed GRBs. If a detection is made, we wish to alert the community to allow for follow up observations by satellite and ground based observatories.

\section{References}

[1] E. Waxman, Phys. Rev. Lett. 75 (1995) 386 [astro-ph/9505082].

[2] IceCube Collaboration, M. G. Aartsen, et al., Science 342 (2013) 1242856 [arXiv:1311.5238].

[3] The Gamma-ray Coordinates Network. http://gcn.gsfc.nasa.gov/

[4] AMANDA Collaboration, J. Ahrens, et al., Nucl. Istrum. and Meth. A 524 (2004) 169 [astro-ph/0407044].

[5] GRBweb. http://grbweb.icecube.wisc.edu/

[6] W. Paciesas, et al., ApJS 199 (2012) 18 [arXiv:1201.3099].

[7] A. Goldstein, et al., ApJS 199 (2012) 19 [arXiv:1201.2981].

[8] R. Aptekar, et al., Space Sci. Rev. 71 (1995) 265.

[9] K. Yamaoka, et al., Publ. Astron. Soc. Japan 61 (2009) 1117 [arXiv:0906.3515].

[10] N. Gehrels, et al., ApJ 611 (2004) 1005 [astro-ph/0405233].

[11] C. Winkler, et al., Astron. Astrophys. 411 (2003) L1.

[12] J. Kent, Journal of the Royal Statistical Society B 44 (1982) 71.

[13] G. Feldman, R. Cousins, Phys. Rev. D 57 (1998) 3873 [physics/9711021].

[14] IceCube Collaboration, M. G. Aartsen, et al., ApJ 805 (2015) L5 [arXiv:1412.6510].

[15] M. Ahlers, et al., Astroparticle Physics 35 (2011) 87 [arXiv:1103.3421].

[16] E. Waxman, J. Bahcall, Phys. Rev. Lett. 78 (1997) 2292 [astro-ph/9701231].

[17] M. W. E. Smith, et al., Astroparticle Physics 45 (2013) 56 [arXiv:1211.5602].

[18] IceCube GRB Limit Tool. http://icecube.wisc.edu/science/tools/grblimits 